\title{
ЭКОНОМИКО-МАТЕМАТИЧЕСКИЕ МОДЕЛИ
}

УДК 336. 004+519.7

\author{
А.Н. Бирюков ${ }^{1}$ \\ Стерлитамакский филиал \\ Башкирского государственного университета,
}

2. Стерлитамак, Россия

О.И. Глущенко

Восточная экономико-юридическая гуманитарная академия, 2. Уфа, Россия

\section{КОРРЕКТНАЯ ПОСТАНОВКА ОБРАТНЫХ ЗАДАЧ \\ ВОССТАНОВЛЕНИЯ МНОГОМЕРНЫХ ФУНКЦИЙ ЧЕРЕЗ НЕЙРОСЕТЕВЫЕ МОДЕЛИ В БЮДЖЕТНОМ АДМИНИСТРИРОВАНИИ}

\begin{abstract}
Аннотация. Объектом рассмотрения настоящей работы является весьма глубокое проникновение требований эффективного обучения нейросетей в алгоритмы предпроцессорной обработки. Разработан метод оценки адекватности нейросетевых моделей в отсутствии каких-либо априорных сведений о законе распределения шумов в данных. Именно это и является научной новизной данной статьи, т. к. данный метод позволяет взаимосвязано управлять качеством предпроцессорной обработки финансовых данных и качеством их аппроксимации в нейросети для бюджетных органов. Предлагается рассматривать класс задач бюджетного администрирования, для которого зашумление данных достаточно большое, поэтому необходимость регуляризации задач восстановления гиперповерхности - необходимая предпосылка эффективности гибридных моделей. В частности указывается, что игнорировать некорректность постановки задачи нельзя. Для ее преодоления имеются два пути: введение обратной задачи в класс корректных (условно корректных по Тихонову) путем привлечения дополнительной информации об искомом решении $Z(\vec{X})$, либо управление классическими алгоритмами решения некорректно поставленных задач. Оба пути основаны на достижениях главным образом отечественных ученых. Существующие регуляризирующие алгоритмы используют исходную базу данных как некоторую внешнюю неизменную «данность». В статье на содержательном практическом примере предложен системный подход к проблеме обеспечения устойчивости нейросетевого отображения при восстановлении гиперповерхности $Y(\vec{X})$ с сильным зашумлением данных на основе теории регуляризации по А.Н. Тихонова, а также байесовского подхода. Суть этого метода состоит в том, что для обеспечения состоятельности алгоритма регуляризации обратной задачи по А.Н. Тихонову база исходных данных не используется как некоторая «застывшая» категория, а подвергается предобработке (структурированию) с использованием общесистемных законов кибернетики (закона энтропийного равновесия открытой системы, неполного подавления дисфункций структурированной системы, резервирования). Таким образом, цель исследования достигнута - суть предполагаемого подхода детализирована и реализована в практической концепции «регуляризация» в данном исследовании с помощью операций алгоритма при построении нейросетевой модели.
\end{abstract}

Ключевые слова: доходная часть бюджета; бюджетополучатели; нейросетевая модель (НСM); интерпретация; налоговые и неналоговые доходы; сеть регуляризации. 


\section{1. Актуальность темы исследования}

Актуальной проблемой остается совершенствование технологии планирования доходной части бюджета любого уровня. Современная деятельность бюджетных органов в условиях кризиса мировой экономики характеризуется неопределенностью внешней и внутренней среды. Результатом этих тенденций является разброс выходных параметров экономической деятельности организаций любого уровня собственности, что во многих случаях предопределяет высокий риск точности бюджетных поступлений. Поэтому переход к математической формализации этапов принятия решений наталкивается на ряд трудностей, связанных с проблемой моделирования плохо формализуемых систем. Одна из таких трудностей в обратных задачах восстановления зависимостей (интерпретации), которые относятся к классу некорректных (или плохо определенных) - это обеспечение устойчивости решения в сложных условиях моделирования.

В работе авторов $[1,3,12]$ была сформулирована концепция комбинированного системного-синергетического-информационного подхода для разработки экономико-математического базиса создания гибридной модели прогноза и принятия решений, включающий в себя нейросетевую модель аппроксимации динамической базы данных, оптимизационную модель оценки эффективности деятельности бюджетополучателей, вероятностную модель оценки риска выхода погрешности прогноза за назначенный уровень.

\footnotetext{
${ }^{1}$ Бирюков Александр Николаевич - доктор экономических наук, доцент, профессор кафедры экономической теории и анализа Стерлитамакского филиала Башкирского государственного университета, г. Стерлитамак, Россия, (453103, Республика Башкортостан, г. Стерлитамак, пр. Ленина, 49); e-mail: biryukov_str@mail.ru.

2 Глущенко Ольга Ивановна - кандидат экономических наук, доцент, докторант Восточной экономикоюридической гуманитарной академии, г. Уфа, Россия, (450054, Республика Башкортостан, г. Уфа, ул. проспект Октября, 71/3); e-mail: Olga.glushhenko@mail.ru.
}

В данной статье предложены новые идеи, основанные на методах нелинейной динамики по управлению качеством нейросетевых моделей, доведенные до конкретных алгоритмов и апробированы на реальных данных бюджетополучателя $[9,10]$.

\section{2. Степень изученности проблемы}

2.1. Общий регуляризирующий оператор Тихонова

«Ядром» всех моделей бюджетного администрирования является задача восстановления «обобщенной производственной функции», скрытой в данных.

Такие задачи восстановления (они называются также задачами интерпретации [2]) относятся к классу обратных задач моделирования, поскольку по известному «следствию» (входным данным) надо восстановить «причину» - неизвестную нелинейную гиперповерхность $Y(\vec{x})$, описывающую закономерности «вход-выход» исследуемого объекта (процесса). Другими словами, речь идет о выявлении скрытых свойств изучаемых объектов.

Для задач интерпретации (восстановления) показано, что задача о минимизации стабилизатора на множестве с ограничениями типа неравенств может быть редуцирована к классической задаче на условный экстремум с ограничением вида равенств (метод Лагранжа) [2]. Такая задача, значительно более удобная для численного решения на ЭВМ, формулируется следующим образом. Пусть $\lambda>0$ числовой параметр. Выражение:

$$
J_{\lambda}(z) \equiv \rho_{\tilde{U}}^{2}(A z, \tilde{u})+\lambda \Omega(z), z \in Z^{*}
$$

называется сглаживающим функционалом Тихонова для задачи интерпретации. Рассмотрим задачу

$$
z^{(\lambda)}=\operatorname{arginf} J_{\lambda}(z), \rho_{\tilde{u}}^{2}\left(A z^{\lambda}, \tilde{u}\right)=\delta^{2},
$$

где второе условие служит для алгоритмического выбора $\lambda$. 
Задача (2) в самом деле допускает более простой алгоритм решения. Для всякого $\lambda>0$ элемент $z^{(\lambda)}$ может быть (обычно однозначным образом) найден каким-либо прямым методом безусловной минимизации $J_{\lambda}$; уравнение (2) есть обычное трансцендентное уравнение $\varphi(\lambda)-\delta^{2}=0$ с алгоритмически определенной левой частью, и оно может быть решено любым из известных методов на ЭВМ; определив отсюда $\lambda=\lambda(\delta)$, находим и $z_{\delta}=z^{(\lambda(\delta))}$. Использование указанного уравнения для определения значения параметра $(\lambda)$ обычно называют методом «невязки» (невязка $\varphi(\lambda))[6,15]$.

Для замыкания задачи восстановления гиперповерхности $\Gamma(x)$ с использованием сглаживающего функционала Тихонова применительно к нейросетевому моделированию «ядра» модели следует определить $\delta$. В качестве $\delta$ предлагается выбирать константу Липшица $L$ как меру неоднородности данных, косвенно зависящую от меры зашумления:

$$
\delta \equiv L=\max _{i \in \overline{1, N}} \frac{\left\|y_{2}-y_{1}\right\|_{E_{n}}}{\left\|\vec{x}_{2}-\vec{x}_{1}\right\|_{E_{n}}},
$$

где $\vec{x}_{2}, \vec{x}_{1}-$ две достаточно близкие точки в базе данных, а $y_{1}, y_{2}$ - соответствующие им значения выхода модели:

$$
\left\|\vec{x}_{2}-\vec{x}_{1}\right\|_{E_{n}} \leq r, r>0 .
$$

Как показано, некоторые обобщеннокорректные постановки обратных задач при самых общих предположениях относительно оператора А и искомого решения $z$ и независимо от класса задачи связаны с конструкцией сглаживающего функционала Тихонова. Оказывается, что задача о минимизации этого параметрического функционала порождает целое семейство регуляризирующих операторов (РО), зависящих от выбора параметра $\lambda$.

В частности, для задач интерпретации (восстановления), описываемых операторным уравнением $A z=u$, существует се- мейство функций $\lambda=\lambda(\delta)$, таких, что экстремаль сглаживающего функционала $z^{\lambda(\delta)}$ сходится в метрике пространства $Z$ при $\lambda \rightarrow 0$ к единственному точному решению операторного уравнения.

Если решение соответствующего операторного уравнения не единственно, то для любой из указанных зависимостей $\lambda=\lambda(\delta)$ имеет место сходимость $\mathrm{z}^{\lambda(\delta)} \kappa$ $\Omega$-нормальному решению [6].

Определение. Любой алгоритм минимизации сглаживающего функционала, построенного для обратной задачи, при заданном значении меры погрешности (или допуска) $\delta$ и каком-либо выборе зависимости $\lambda=\lambda(\delta)$, удовлетворяющей принципу регуляризации (или обеспечивающей принадлежность $z^{\lambda(\delta)}$ множеству допустимых значений), называется общим регуляризирующий оператором (РО) Тихонова.

Для реализации общего регуляризирующего оператора Тихонова нужно решить следующие задачи [6].

1. Сформулировать алгоритм вычисления «прямых эффектов» т.е. «невязки» $\rho_{\tilde{u}}^{2}\left(A z^{\lambda}, \tilde{u}\right)$. Для обратных задач это функционал качества, вообще говоря, более общего вида, чем «невязка». Отметим, что расчет прямых эффектов осуществляется многократно в ходе решения обратной задачи и занимает обычно основную долю машинного времени. Ввиду этого следует позаботиться о том, чтобы соответствующий алгоритм был по возможности максимально экономичным.

2. Выбрать стабилизатор $\Omega(z)$, учитывая имеющуюся априорную информацию о решении.

3. Следует выбрать способ согласования параметра $\lambda \mathrm{c} \delta: \lambda=\lambda(\delta)$ в соответствии с принципом регуляризации.

4. Выбрать стратегию минимизации сглаживающего функционала. Отметим, что в этом отношении РО не отличается от алгоритмов, вытекающих из других корректных 
вариационных постановок и связан с использованием известных алгоритмов минимизации; вместе с тем структура сглаживающего функционала приводит к достаточно экономичной для ряда задач стратегии.

Один из способов согласования параметра $\lambda$ с величиной $\delta$ по «невязке» был упомянут, и он является элементом постановки задачи (2). Поскольку задача решается на алгоритмически вводимом множестве корректности, о близости $z^{\lambda(\delta)}$ к точному решению можно судить по близости наблюдаемого и рассчитанного эффектов, что и делают при таком выборе регуляризованного приближения. Этот алгоритм является общим для задач всех типов, если для них известна величина $\delta$.

\section{2. Регуляризация радиально-базис- ные сетей (RBF-сетей)}

$\mathrm{B}$ теории регуляризации RBF-сетей используются специфические свойства этих сетей [7]:

- Имеется только один скрытый слой (рис. 1), число нейронов, в котором равно числу сигналов, т.е. числу $N$ примеров в данных.

- Активационные функции в узлах скрытого слоя - это функции Грина

$G\left(\vec{x}, \vec{x}_{i}\right)$ либо их частный случай радиальные базисные функции $\varphi\left\|\vec{x}_{i}-\vec{x}\right\|$, где $\vec{x}_{i}-$ центр для функций Грина; $\vec{x}$ - текущее значение ее аргумента.

- В Выходной слой в случае одномерного выхода $\left(y \in R^{1}\right)$ представляет собой нейрон, выходом которого служит линейная взвешенная сумма всех выходов узлов промежуточного слоя.

- В случае если в качестве функции Грина используется многомерная функция Гаусса (33), то она обладает нужным для регуляризации свой- ством непрерывной дифференцируемости, включая вторые производные по своим аргументам.

Запишем функционал сглаживания А.Н. Тихонова (1) в виде:

$$
F_{\lambda}(z)=E_{s}(z)+E_{c}(z) .
$$

Здесь первое слагаемое, обозначаемое $E_{s}(F)$, описывает стандартную ошибку (расстояние между желаемым откликом $d_{i}$ и фактическим выходным сигналом нейросети $y_{i}$, для примера обучения $\left.i=1,2, \ldots, N\right)$. В частности, можно определить:

$$
E_{s}(F)=\frac{1}{2} \sum_{i=1}^{N}\left(d_{i}-y_{i}\right)^{2}=\frac{1}{2} \sum_{i=1}^{N}\left[d_{i}-F\left(\vec{x}_{i}\right)\right]^{2},
$$

где $F\left(\vec{x}_{i}\right) \equiv \hat{Y}\left(\vec{x}_{i}, W\right)$ - нейросетевое отображение в задаче интерполяции.

Второе слагаемое, обозначенное $E_{c}(F)$, в классической записи функционала А.Н. Тихонова равно:

$$
E_{c}(F)=\lambda \Omega(F(\vec{x})),
$$

должно зависеть от «геометрических» свойств функции аппроксимации нейросети $F(\vec{x})$.

Учитывая свойства гладкости функции

Грина $G\left(\vec{x}, \vec{x}_{i}\right)$, упомянутые выше, можно положить:

$$
E_{c}(F)=\frac{1}{2} \lambda\|D F\|_{L_{2}}^{2},
$$

где $D$ - линейный дифференичальный опеpaтор. Априорная информация о форме решения (т.е. о функции отображения $F(\vec{x})$ ), включенная в дифференциальный оператор $D$, обеспечивает его зависимость от конкретной задачи. Оператор $D$ иногда еще называют стабилизатором, т. к. в задаче регуляризации он стабилизирует решение, делая его гладким и, таким образом, удовлетворяющим свойству непрерывности.

Символ $\|\cdot\|$ в выражении (8) обозначает норму в функциональном пространстве, к которому принадлежит $D F(\vec{x})$. При обычных условиях используемое здесь функцио- 
нальное пространство является гильбертовыл пространством $L_{2}$, состоящим из всех действительных функций $f(\vec{x}), \vec{x} \in R^{n}$, для которых норма $\|f(\vec{x})\|^{2}$ является интегрируемой по Лебегу. Используемая здесь функция $f(\vec{x})$ обозначает фактическую функцию, описывающую моделируемый экономичный процесс, отвечающий за генерацию пар примеров обучения $\left\{\left(\vec{x}_{i}, d_{i}\right)\right\}_{i=1}^{N}$.
Величиной, которую требуется минимизировать в теории регуляризации, является

$$
\begin{aligned}
& E(F)=E_{s}(F)+\lambda E_{c}= \\
& =\frac{1}{2} \sum_{i=1}^{N}\left[d_{i}-F\left(\vec{x}_{i}\right)\right]^{2}+\frac{1}{2} \lambda\|D F\|^{2},
\end{aligned}
$$

где $\lambda$ - положительное действительное число, называемое параметром регуляризации; $E(F)$ - функиионал Тихонова. Функционал отображает функции (определенные в соот-

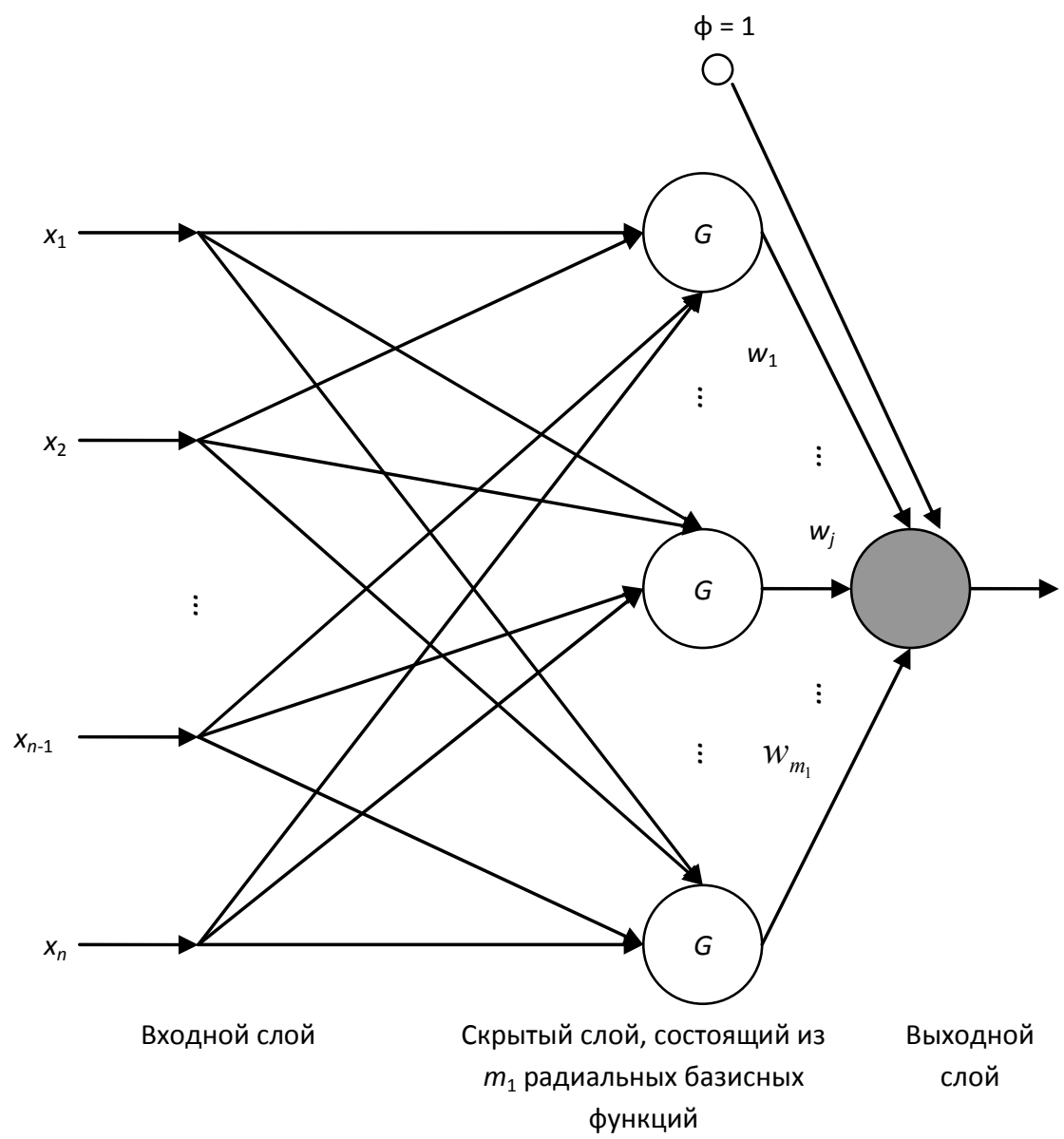

Pис. 1. Сеть регуляризацчии в терминах радиальных базисных функичй (RBF-сети)

Bulletin of Ural Federal University. Series Economics and Management. 2017. Vol. 16. No 2. PP. 227-245 
ветствующем функциональном пространстве) на ось действительных чисел.

$$
E(F): L_{2} \rightarrow R^{1}
$$

Аргминимум функционала Тихонова $E(F)$ (т.е. решение задачи регуляризации) обозначается $F_{\lambda}(\vec{x})$ :

$$
J_{\lambda}(\vec{x}): \arg \min _{F} E(F) .
$$

В некотором смысле параметр регуляризации $\lambda$ можно рассматривать как индикатор достаточности данного набора данных для определения решения $J_{\lambda}(\vec{x})$. В частности, крайний случай, $\lambda \rightarrow 0$, означает, что задача является безусловной и имеет решение $J_{\lambda}(\vec{x})$, целиком зависящее от примеров. Другой крайний случай, $\lambda \rightarrow \infty$, предполагает, что самого априорного ограничения на гладкость, представленного дифференциальным оператором $D$, достаточно для определения решения $J_{\lambda}(\vec{x})$. Это может указывать также на недостаточное количество примеров. В практических приложениях параметр регуляризации $\lambda$ принимает некоторое среднее значение между этими двумя крайними случаями. Этим определяется влияние на решение $J_{\lambda}(\vec{x})$ как априорной информации, так и данных обучающей выборки. Таким образом, слагаемое регуляризации $E_{c}(F)$ представляет собой функцию штрафа за сложность модели, влияние которой на окончательное решение определяется параметром регуляризации $\lambda$.

В [7] показано, что с помощью применения тождества Грина к выражению дифференциала Фреше $d E_{c}(F, h)$ из (9) и затем использования уравнения Эйлера - Лагранжа и функции Грина $G(\vec{x}, \vec{\xi})$ можно получить уравнение для выбора аппроксимирующего восстанавливающего оператора $F(\vec{x})$ многомерную функцию $Y(\vec{X})$ :

$$
L F(\vec{x})=\phi(\vec{x}),
$$

где $\varphi(\vec{x})$ непрерывная или кусочно-непрерывная функция аргумента $\vec{x} \in R^{n} ; L-$ линейный дифференциальный оператор.
Решением дифференциального уравнения (12) является непрерывная или кусочно-непрерывная функция $F(\vec{x})$ аргумента $\vec{x} \in R^{n}$ :

$$
F(\vec{x})=\int_{R^{n}} G(\vec{x}, \vec{\xi}) \varphi(\xi) d \xi,
$$

где $G(\vec{x}, \vec{\xi})-$ функция Грина для линейного оператора дифференцирования $L$ [2]; $\xi$ - переменная интегрирования.

Теперь оговорим свойства функции Грина $G(\vec{x}, \vec{\xi})$, которая в RBF-сетях используется в качестве активационной:

1. Для фиксированного аргумента $\vec{\xi}$ $G(\vec{x}, \vec{\xi})$ является функцией от $\vec{x}$ и удовлетворяет граничным условиям.

2. Во всех точках, исключая $\vec{x}=\vec{\xi}$, все производные от $G(\vec{x}, \vec{\xi})$ по переменной $\vec{x}$ являются непрерывными, количество производных определяется порядком оператора $L$.

3. По переменной $\vec{x}$ функция $G(\vec{x}, \vec{\xi})$ удовлетворяет уравнению в частных производных:

$$
L G(\vec{x}, \vec{\xi})=0,
$$

всюду, кроме точки $\vec{x}=\vec{\xi}$, где $G(\vec{x}, \vec{\xi})$ имеет особенность. Это значит, что функция $G(\vec{x}, \vec{\xi})$ удовлетворяет уравнению в частных производных в смысле распределения:

$$
L G(\vec{x}, \vec{\xi})=\delta(\vec{x}-\vec{\xi}),
$$

где $\delta(\vec{x}-\vec{\xi})$ - дельта-функция Дирака с центром в точке $\vec{x}=\vec{\xi}$.

\section{3. Методика исследования}

\section{1. Решение задачи регуляризации}

Следуя [2], запишем уравнение Эйлера - Лагранжа для функционала Тихонова $E(F(\vec{x})$. Оно является необходимым условием существования экстремума функционала Тихонова в точке $F_{\lambda}(\vec{x})$ : 


$$
\tilde{D} D_{\lambda} J(\vec{x})=\frac{1}{\lambda} \sum_{i=1}^{N}\left[d_{i}-F\left(\vec{x}_{i}\right)\right] \delta\left(\vec{x}-\vec{x}_{i}\right) .
$$

Здесь $\widetilde{D}$ - оператор, сопряженный с $D$.

Тогда с учетом $L=\widetilde{D} D$ и (12) можно записать решение уравнения Эйлера - Лагранжа в виде [2]:

$$
J_{\lambda}(\vec{x})=\frac{1}{\lambda} \sum_{i=1}^{N}\left[d_{i}-F\left(\vec{x}_{i}\right)\right] G\left(\vec{x}, \vec{x}_{i}\right) .
$$

Выражение (16) означает, что аргминимум функционала Тихонова регуляризации является линейной суперпозицией $N$ функций Грина. Векторы $\vec{x}_{i}$, представляют собой центры разложения, а веса $\left[d_{i}-F\left(\vec{x}_{i}\right)\right] / \lambda-$ коэффичиенты разложения решения по базиснылм функциям Грина.

Другими словами, решение задачи регуляризации лежит в $N$-мерном подпространстве пространства гладких функций, а множество функций Грина $\left\{G\left(\vec{x}, \vec{x}_{i}\right)\right\}$, с центром в $\vec{x}_{i}, i=1,2, \ldots, N$, определяется базис этого подпространства [2].

Коэффициенты разложения в (16) являются, во-первых, линейными относительно ошибки оценки, определенной как разность между желаемым откликом $d_{i}$ и соответствующим выходным сигналом нейросети $F\left(\vec{x}_{i}\right)$, и, во-вторых, обратно пропорциональными параметру регуляризации $\lambda$.

\section{2. Алгоритм определения коэффи- циентов разложения}

Следующим вопросом является определение коэффициентов разложения в выражении (16). Положим синоптические веса нейронов положить равными:

$$
w_{i}=\frac{1}{\lambda}\left[d_{i}-F\left(\vec{x}_{i}\right)\right], i=1,2, \ldots, N .
$$

Тогда выражение минимизирующего решения (16) можно упростить, т.е. явно выразить через функции Грина:

$$
J_{\lambda}(\vec{x})=\frac{1}{\lambda} \sum_{i=1}^{N} w_{i} G\left(\vec{x}, \vec{x}_{i}\right) .
$$

Вычисляя $J_{\lambda}(\vec{x})$ в точке $\vec{x}_{j}, j=1,2, \ldots, N$, получим:

$$
J_{\lambda}\left(\vec{x}_{j}\right)=\frac{1}{\lambda} \sum_{i=1}^{N} w_{i} G\left(\vec{x}_{j}, \vec{x}_{i}\right), j=1,2, \ldots, N .
$$

Введем следующие обозначения:

$$
\begin{aligned}
\vec{J}_{\lambda} & =\left[J_{\lambda}\left(\vec{x}_{1}\right), F_{\lambda}\left(J_{2}\right), \ldots, J_{\lambda}\left(\vec{x}_{N}\right)\right]^{T}, \\
\vec{d} & =\left[d_{1}, d_{2}, \ldots, d_{N}\right]^{T}, \\
G & =\left[\begin{array}{cccc}
G\left(\vec{x}_{1}, \vec{x}_{1}\right) & G\left(\vec{x}_{1}, \vec{x}_{2}\right) & \ldots & G\left(\vec{x}_{1}, \vec{x}_{N}\right) \\
G\left(\vec{x}_{2}, \vec{x}_{1}\right) & G\left(\vec{x}_{2}, \vec{x}_{2}\right) & \ldots & G\left(\vec{x}_{2}, \vec{x}_{N}\right) \\
\ldots & \ldots & \ldots & \ldots \\
G\left(\vec{x}_{N}, \vec{x}_{1}\right) & G\left(\vec{x}_{N}, \vec{x}_{2}\right) & \ldots & G\left(\vec{x}_{N}, \vec{x}_{N}\right)
\end{array}\right], \\
\vec{w} & =\left[w_{1}, w_{2}, \ldots, w_{N}\right]^{T} .
\end{aligned}
$$

Теперь (17) и (18) можно переписать в матричной форме:

$$
\begin{aligned}
w & =\frac{1}{\lambda}\left[\vec{d}-\vec{J}_{\lambda}\right], \\
\vec{J}_{\lambda} & =G \vec{w} .
\end{aligned}
$$

Подставляя выражение для $J_{\lambda}(\vec{x})$ из (25) и (24) и переставляя слагаемые, получим:

$$
(G+\lambda I) \vec{w}=\vec{d},
$$

где $I$ - единичная матрица размерности $N \times N$. Матрица $G$ называется матрицей Грина. Оператор дифференцирования $L$, определяемый выражением $L=\widetilde{D} D$, является самосопряженныл. Это значит, что оператор, сопряженный $L$, равен ему самому. Отсюда следует, что ассоциированная функция Грина $G\left(\vec{x}, \vec{x}_{i}\right)$ является симметричной, т.е.

$$
G\left(\vec{x}_{i}, \vec{x}_{j}\right)=G\left(\vec{x}_{j}, \vec{x}_{i}\right) \forall i, j .
$$

Уравнение (27) означает, что позиции двух точек $\vec{x}_{i}$ и $\vec{x}_{i}$ можно поменять местами, при этом значение функции Грина $G\left(\vec{x}_{j}, \vec{x}_{i}\right)$ не изменится. Аналогично, матрица Грина, определяемая выражением (22), является симметричной, т.е.

$$
G T=G \text {. }
$$

Теперь можно применить теорему Мичелли [6] об интерполяции, в контексте ма- 
трицы интерполяции $B$. Во-первых, заметим, что матрица Грина $G$ играет в теории регуляризации роль, аналогичную той, которую матрица $B$ играет в теории интерполяции на основе сетей RBF. Обе матрицы, $B$ и $G$, являются симметричными и имеют размерность $N \times N$. Следовательно, можно утверждать, что матрица $G$ для некоторых классов функций Грина так же, как и матрица $J$, является положительно определенной при условии, что точки $\vec{x}_{1}, \ldots \vec{x}_{N}$ различны. Классы функций Грина, охватываемые теоремой Мичелли [6], включают обратные параболические функции и функции Гаусса, но не включают параболические функции. На практике всегда можно выбрать значение $\lambda$, достаточно большое для обеспечения положительной определенности матрицы $(G+\lambda I)$ и, таким образом, для возможности ее обращения. А это, в свою очередь, значит, что система линейных уравнений (26) имеет единственное решение, определяемое следующим образом [7]:

$$
\vec{w}=(G+\lambda I)^{-1} \vec{d} .
$$

Таким образом, выбрав оператор дифференцирования $D$ и имея набор функций Грина $G\left(\vec{x}, \vec{x}_{i}\right)$, где $i=1,2, \ldots, N$, соотношение (29) можно использовать для получения вектора весов $\vec{w}$, соответствующего вектору желаемого отклика $\vec{d}$ и данному значению параметра регуляризации $\lambda$.

В заключение можно утверждать, что решение задачи регуляризации для RBFсетей задается разложением (18), где $G\left(\vec{x}, \vec{x}_{i}\right)$ функция Грина для самосопряженного оператора $L=\widetilde{D} D ; w_{i}-i$-й элемент вектора весов $\vec{w}$. Уравнение (18) означает следующее [7]:

- Регуляризационный подход эквивалентен разложению решений в терминах множества функций Грина, характеристики которых зависят от принятой формы оператора дифференцирования $D$ в стабилизаторе Ти- хонова и соответствующих граничных условий.

- Количество функций Грина, используемых в разложении, равно количеству примеров, используемых при обучении.

Характеристика функции Грина $G\left(\vec{x}, \vec{x}_{i}\right)$ для заданного центра $\vec{x}_{i}$ зависит только от формы стабилизатора $D$ (согласно априорному предложению касательно искомого отображения «вход-выход»). Если стабилизатор $D$ является инвариантным к преобразованиям, то функция Грина $G\left(\vec{x}, \vec{x}_{i}\right)$ с центром в $x_{i}$ будет зависеть только от разности между аргументами $\vec{x}$ и $\vec{x}_{i}$, т.е.

$$
G\left(\vec{x}, \vec{x}_{i}\right)=G\left(\vec{x}-\vec{x}_{i}\right) .
$$

Если же стабилизатор $D$ инвариантен как к преобразованиям, так и к поворотам, то функция Грина $G\left(\vec{x}, \vec{x}_{i}\right)$ будет зависеть только евклидовой нормы вектора разности $\left(\vec{x}-\vec{x}_{i}\right)$. То есть

$$
G\left(\vec{x}, \vec{x}_{i}\right)=G\left(\left\|\vec{x}-\vec{x}_{i}\right\|\right) .
$$

При этих условиях функция Грина должна быть радиальной базисной функцией. В таком случае решение задачи регуляризации (18) принимает следующую частную форму [7]:

$$
J_{\lambda}(\vec{x})=\frac{1}{\lambda} \sum_{i=1}^{N} w_{i} G\left(\left\|\vec{x}-\vec{x}_{i}\right\|\right) .
$$

Решение (32) определяется пространство линейных функций, зависящее от известных точек данных и с учетом евклидова расстояния.

Решение, описанное выражением (32), называется строгой интерполяиией, т. к. для интерполяции функции $F(\vec{x})$ используются все $N$ точек, доступных для обучения. Однако при этом важно отметить, что это решение регуляризировано с помощью определения (29) для вектора весов $\vec{w}$. Только при достижении параметров регуляризации значения, равного нулю, эти два решения становятся идентичными. 


\section{3. Многомерные функции Гаусса}

Функция Грина $G\left(\vec{x}, \vec{x}_{i}\right)$, линейный дифференциальный оператор $D$, которой инвариантен к трансформациям и вращению и удовлетворяет условию (31), представляют на практике большой интерес. Примером такой функции Грина является многомерная функиия Гаусса, определяемая следующим образом [7]:

$$
G\left(\vec{x}, \vec{x}_{i}\right)=\exp \left(-\frac{1}{2 \sigma_{i}^{2}}\left\|\vec{x}-\vec{x}_{i}\right\|\right) .
$$

Определяя функцию Грина $G\left(\vec{x}, \vec{x}_{i}\right)$ в специальном виде (33), регуляризируемое решение (32) принимает форму линейной суперпозиции многомерных функций Гаусса:

$$
F_{\lambda}(\vec{x})=\sum_{i=1}^{N} w_{i} \exp \left(-\frac{1}{2 \sigma_{i}^{2}}\left\|\vec{x}-\vec{x}_{i}\right\|\right),
$$

где сами линейные веса $w_{i}$ определяются по формуле (17).

В выражении (34) отдельные слагаемые функции Гаусса, определяющие функцию аппроксимации $F(\vec{x})$, в качестве аргументов содержат разные переменные. Для упрощения изложения в $F(\vec{x})$ часто принимается условие $\sigma_{\mathrm{i}}=\sigma$ для всех $i$, несмотря на то, что определенные таким образом функции имеют несколько ограниченный вид, они остаются универсальными аппроксиматорами [2].

Таким образом, можно сделать обобщающий вывод по методологии обеспечения устойчивости задачи восстановления гиперповерхности с помощью нейросетей RBF-типа для прикладных задач с наиболее сложными условиями моделирования сильным зашумлением данных, отягченным дефицитом наблюдений.

Целесообразно ставить задачу восстановления как задачу строгой интерполяции с использованием $N$ доступных обучению примеров и выбору RBF-сетей с радиальными базисными функциями, удовлетворяющими методу регуляризации Тихоно- ва. При этом интерполирующий оператор $J_{\lambda}(\vec{x})$ находится в виде линейной комбинации (разложения) базисных функций Грина, в частности многомерных функций Гаусса, а существование решения матричного уравнения (29) для синоптических весов гарантируется положительной определенностью матрицы Грина $G$ и, соответственно матрицы $(G+\lambda I)$, где $\lambda$ - параметр регуляризации $(\lambda>0)$. Число радиальных базисных функций (RBF) в разложении, и соответственно число нейронов скрытого слоя равно числу $N$ примеров, т.е. векторов $\vec{x}_{i}$ в данных, что важно в условиях дефицита наблюдений.

\section{4. Оценивание параметров регуля- ризации}

Оценивание параметра регуляризации $\lambda$ для построения регуляризированного решения $J_{\lambda}(\vec{x})$ является нетривиальной задачей [2]. Прежде всего нужно выбрать числовую меру для качества оценки $\lambda$, т.е. нахождения оптимального значения $\lambda^{*}$ либо, чтобы сполна использовать возможности теории регуляризации, необходимо применить столь же принципиальный подход к оценке $\lambda$. Общая идея оценки параметра регуляризации состоит в минимизации среднеквадратической ошибки $S(\lambda)$ между восстанавливаемой нелинейной многомерной функцией $Y\left(\vec{x}_{i}\right)$, «зашитой» в зашумленной базе данных, и расчетными значениями $F_{\lambda}(\vec{x})$ регуляризированного решения на множестве точек $i=\overline{1, N}$.

Главная трудность здесь состоит в том, что восстанавливаемая функция $Y(\vec{x})$ априори неизвестна. Эту трудность можно обойти, если в задаче минимизации для определения $\lambda^{*}$ заменить теоретическое значение $S(\lambda)$ на некоторую приближенную статическую оценку $\hat{S}(\lambda)$, удовлетворяющую требованиями состоятельности и несмещенности: 


$$
\begin{aligned}
& S(\lambda) \rightarrow \hat{S}(\lambda): M[\hat{S}(\lambda)]=M[S(\lambda)] ; \\
& \lim P(|S(\lambda)-\hat{S}(\lambda)|<\eta) \rightarrow 1,
\end{aligned}
$$

где $P(\bullet)$ - вероятность; $\eta>0$ - сколь угодно малое число; $M(\bullet)$ - оператор математического ожидания.

Отметим одну общую особенность выбора $\lambda$, который является нетривиальной задачей, ибо этот выбор должен обеспечить компромисс между следующими двумя противоречивыми моментами:

Грубость решения, определяемая стабилизатором Тихонова $\|D F(\vec{x})\|_{L_{2}}^{2}$.

Недостоверность данных, определяемая слагаемым стандартной ошибки $\sum_{i=1}^{N}\left[d_{i}-F\left(x_{i}\right)\right]^{2}$.

Для реализации разложения регуляризованной функции аппроксимации $J_{\lambda}(\vec{x})$, предоставленной в (32) в терминах функции Грина $G\left(\vec{x}, \vec{x}_{i}\right)$ с центром в точке $\vec{x}_{i}$, можно использовать нейросетевую структуру, показанную на рис. 1. Такие сети называются сетями регуляризации. Эта сеть имеет три слоя. Входной слой состоит из входных узлов, количество которых равно размерности $n_{0}$ вектора входного сигнала $\vec{x}$ (т.е. количеству независимых переменных в задаче). Второй слой является скрытым. Он состоит из нелинейных элементов, которые непосредственно связаны со всеми узлами входного слоя. Для каждой точки данных $\vec{x}_{i}(i=1,2, \ldots, N$, где $N$ - размер множества примеров обучения) существует свой скрытый узел. Функциями активации отдельных узлов скрытого слоя являются функции Грина. Следовательно, выходной сигнал $i$-го нейрона скрытого слоя определяется как $G\left(\vec{x}, \vec{x}_{i}\right)$. Выходной слой состоит из единственного линейного нейрона, связанного со всеми узлами скрытого слоя. Под «линейностью» подразумевается то, что его выход является линейно-взвешенной суммой всех выходных сигналов скры- того слоя согласно (29). Веса выходного слоя являются неизвестными коэффициентами разложения, определяемого в терминах функций Грина и параметра регуляризации $\lambda$.

1. Сеть регуляризации является универсальным аппроксиматором в том смысле, что при большом количестве скрытых элементов она способна довольно хорошо аппроксимировать любую непрерывную функцию на компактном подмножестве из $R^{n}$.

2. Так как схема аппроксимации, вытекающая из теории регуляризации, является линейной относительно неизвестных коэффициентов, то сети регуляризации обладают свойством наилучшей аппроксимации. Это значит, что для неизвестной линейной функции $f$ всегда существует такой набор коэффициентов, который аппроксимирует функцию $f$ лучше любого другого набора.

3. Решение, обеспечиваемое сетью регуляризации, является оптимальным. Под оптимальностью здесь понимается то, что сеть минимизирует функционал, измеряющий удаленность решения от своего истинного значения, представленного примерами обучения.

4. Анализ полученных результатов. Факторный анализ доходной части бюджета по нейросетевой модели

Практически концепция «регуляризация» реализована в нашем исследовании с помощью следующих операций алгоритма при построении НСМ:

- $\quad$ всех процедур предобработки данных (предрегуляризации);

- процедур байесовской регуляризации обучения нейросетей с апостериорной фильтрацией гипотез.

Произведем выбор зависимых и независимых переменных.

Моделируемая (выходная) величина $Y-$ консолидированный доход бюджета муниципального образования (МО) $[9,10]$. 
Выбор каждой из независимых переменных требует подробных комментариев.

Поскольку строится динамическая модель, то в качестве носителя информации о «быстром времени» (с интервалом 1 месяц) выбрана независимая переменная $X_{1}$ - относительное время:

$$
X_{1}=t_{k} / T
$$

где $t$ - текущее время отсчета, измеряемое в месяцах; $T$ - период наблюдения, равный 48 месяцам (2012-2015 гг.) $[13,14]$. Таким образом, диапазон изменения объясняющей переменной $X_{1}$ составляет: $X_{1} \in[0,0208 ; 1]$.

В качестве управляющих воздействий, формирующих поступление финансовых средств в бюджет муниципального образования, в модель введены две объясняющие переменные:

$X_{2}$ - норматив налога на доходы физических лиц, отчисляемого в местный бюджет (НДФЛ), \%;

$X_{3}$ - норматив единого налога на вмененный доход для отдельных видов деятельности, отчисляемого в местный бюджет (ЕНВД), \%

Указанные нормативы устанавливаются местными органами самоуправления и прямо влияют на наполнение бюджета, а также являются обратной связью, косвенно влияющей на доходную часть бюджета МО через формирование микроклимата для малого и среднего бизнеса на территории МО.

Объясняющие переменные $X_{4}, X_{5}$ характеризуют соотношения налоговых и неналоговых доходов:

$X_{4}$ - сумма налоговых доходов, которая представляет собой агрегированный показатель, который включает в себя одиннадцать составляющих: налог на прибыль организаций; налог на доходы физических лиц; акцизы на алкогольную продукцию; акцизы на пиво; единый налог, взимаемый в связи с применением упрощенной системы налогообложения; единый налог на вмененный доход на отдельные видов деятельности; налог на имущество физических лиц; налог на имущество организаций; налог на добычу полезных ископаемых; земельный налог; госпошлина; прочие налоги;

$X_{5}$ - сумма неналоговых доходов, которая тоже является агрегированным показателем, включающим семь компонентов: арендную плату за земли МО; доходы от сдачи в аренду имущества, находящегося в муниципальной собственности; доходы от перечисления части прибыли муниципальных унитарных предприятий; платежи за негативное воздействие на окружающую среду; доходы от оказания платных услуг и др.

Для учета предыстории экономического процесса, т.е. «медленного времени» при формировании «регуляризации», предложено ввести в модель лаговые переменные.

Для выявления значимых лагов была построена автокорреляционная функция временного ряда $Y\left(X_{1}\right)$ которая показала, что наиболее значимыми являются лаги $\tau_{1}=1$ месяц и $\tau_{2}=3$ месяца (или в относительных единицах $\tau_{1}=0,0208$ и $\tau_{2}=0,0624$. С указанными лагами введены переменные:

$$
X_{6, t} \equiv Y_{t-0,0208} ; X_{7, t} \equiv Y_{t-0,0624} \text {. }
$$

То есть значение объясняющей лаговой переменной $X_{6}$ в момент времени $t$ равно значению выходной (зависимой переменной $Y$ ), сдвинутому назад на один временной интервал. Лаговая переменная $X_{7}$ равна значению $Y$, сдвинутому назад на три временных интервала.

Теория и практика нейросетевого моделирования [3-5, 8] показывает, что введение лаговых переменных значительно улучшает прогностические свойства модели. Поскольку наблюдения охватывают достаточно длительный период (3 года), в течение которого изменяется внешняя экономическая среда, введен фактор -

Bulletin of Ural Federal University. Series Economics and Management. 2017. Vol. 16. No 2. PP. 227-245 
$X_{8}$ - средний индекс инфляции, вычисляемый по формуле:

$$
X_{8}=(\text { ИПЦ + ИЦП }) / 2 \text {, }
$$

где ИПЦ - индекс потребительских цен; ИЦП - индекс цен на промышленную продукцию.

Объясняющая переменная $\mathrm{X}_{9}$ несет информацию об управленческих расходах по обслуживанию бюджета, в т.ч. фонд оплаты труда госслужащих муниципального образования.

Таким образом, сформирована база данных для построения нейросетевой прогнозной модели (табл. 1).

Построенная НСМ позволяет не только вычислять прогнозные оценки доходной части бюджета, но и проводить анализ однофакторных зависимостей вида:

$$
\hat{Y}=\varphi\left(X_{j}\right) X_{k}=\text { const, } j \neq k, j=2,9 .
$$

Для этого в табл. 1 базы данных фиксируются значения всех входных факторов $X_{k}$, кроме анализируемого $X_{j}$, и строятся кривые вида (рис. 2). По ним можно судить о характере однофакторной зависимости $\varphi(X)$, вычислять коэффициенты эластичности, $\beta$ и $\Delta$ - коэффициенты.

Если $X_{j} \equiv X_{1}$, то по динамическим кривым $\hat{Y}(t), X_{j}(t),(j \neq 1)$ можно анализировать динамические экономические показатели: рост и прирост, темпы роста и прироста, как самой суммы наполнения бюджета $\hat{Y}$, так и входных факторов модели $X_{2}-X_{9}$, формирующих этот бюджет.

В качестве примера на рис. 3-5 показаны зависимости суммы бюджета $\hat{Y}$ от относительного времени $X_{1}$, от $X_{2}$ - норматива на доходы физических лиц, отчисляемого в местный бюджет в процентах, и от $X_{9}$ - информацию об управленческих расходах по обслуживанию бюджета, в т.ч. фонда оплаты труда госслужащих. В табл. 2 приведены соответствующие результаты расчета по шести параллельным HCM.

Таблица 1

База данных прогнозирования наполнения бюджета за счет налоговых

\begin{tabular}{|c|c|c|c|c|c|c|c|c|c|c|c|c|}
\hline 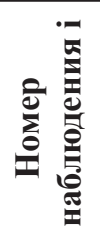 & Год & $\stackrel{\bar{E}}{\stackrel{E}{E}}$ & $X_{1}$ & $X_{2}$ & $X_{3}$ & $X_{4}$ & $X_{5}$ & $X_{6}$ & $X_{7}$ & $X_{8}$ & $X_{9}$ & $\boldsymbol{Y}$ \\
\hline $\begin{array}{c}1 \\
\ldots . . \\
48 \\
\end{array}$ & $\begin{array}{c}2012 \\
\ldots \ldots \\
2015\end{array}$ & $\begin{array}{c}\mathrm{I} \\
\ldots \\
\mathrm{XII}\end{array}$ & $\begin{array}{c}0,02 \\
\ldots \\
1,0\end{array}$ & $\begin{array}{r}0,6 \\
0,37\end{array}$ & $\begin{array}{l}\ldots \\
\ldots\end{array}$ & $\begin{array}{l}\cdots \\
\ldots \\
\ldots\end{array}$ & $\begin{array}{l}\cdots \\
\ldots \\
\ldots \\
\end{array}$ & $\begin{array}{l}\cdots \\
\ldots \\
\ldots\end{array}$ & $\begin{array}{l}\cdots \\
\ldots \\
\ldots\end{array}$ & $\begin{array}{l}17,2 \\
6,82 \\
\end{array}$ & $\begin{array}{c}8,41 \\
13,47\end{array}$ & $\begin{array}{c}57 \\
78,59\end{array}$ \\
\hline
\end{tabular}
и неналоговых поступлений МО

Таблица 2

\begin{tabular}{|c|c|c|c|c|c|c|}
\hline$X_{1}$ & $Y$ HC1 & $Y \mathrm{HC2}$ & $Y \mathrm{HC3}$ & Y HC4 & $Y$ HC5 & Y HC6 \\
\hline 0,2 & 83,19048 & 71,05648 & 81,31596 & 72,61283 & 74,51833 & 68,92414 \\
\hline$\ldots \ldots$ & $\ldots$ & $\ldots \ldots$ & 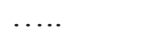 & $\ldots$ & $\ldots \ldots$ & $\ldots \ldots$ \\
\hline 13,47 & 81,23087 & 69,41758 & 81,90119 & 74,33774 & 78,57992 & 84,85222 \\
\hline
\end{tabular}

Расчет однофакторных зависимостей $Y(X), j=1,2,9$ 
Приведем некоторые оценки по факторному анализу.

1. На рис. 3 видно, что при фиксированном значении всех факторов, кроме временного $X_{1}$, сумма бюджета растет практически линейно. Средний (по 6 НСМ и по интервалу времени) прирост к концу наблюдений (точка $t=48$ ) составляет:

$\Delta \hat{Y}_{\mathrm{cp}}=14,41$ млн руб.;

Средняя скорость прироста равна:

$\Delta \hat{Y}_{\text {cp }} / \Delta \mathrm{t}=14,41$ млн руб./19,2 месяцев $=$ $=0,758$ млн руб./месяц.

Замечание. Указанная на рис. 3 линейная зависимость по $\hat{Y}$ от времени является мнимой (виртуальной), ибо она получена при «замораживании» всех остальных факторов $X_{2}, X_{3}, \ldots, X_{9}$, введенных в НСМ. Ре- альная же динамическая кривая при одновременном действии всех факторов имеет сложный вид, показанный на рис. 2. Она весьма далека от идеализированной линейной кривой. И, главное, реальная кривая имеет не растущий, а падающий в среднем характер и дает в точке прогноза отрицательный прирост:

$$
\begin{aligned}
& \Delta \hat{Y}=(49,44-57,22) \pm 3,84= \\
& =(-7,78 \pm 3,84) \text { млн руб. }
\end{aligned}
$$

Таким образом, НСМ дает более пессимистическую, но реальную и достаточно достоверную оценку прироста при действии всех факторов.

2. Кривая зависимости расчетной суммы $\hat{Y}$ доходной части от норматива на доходы физических лиц $X_{2}$ (рис. 4) показыва-

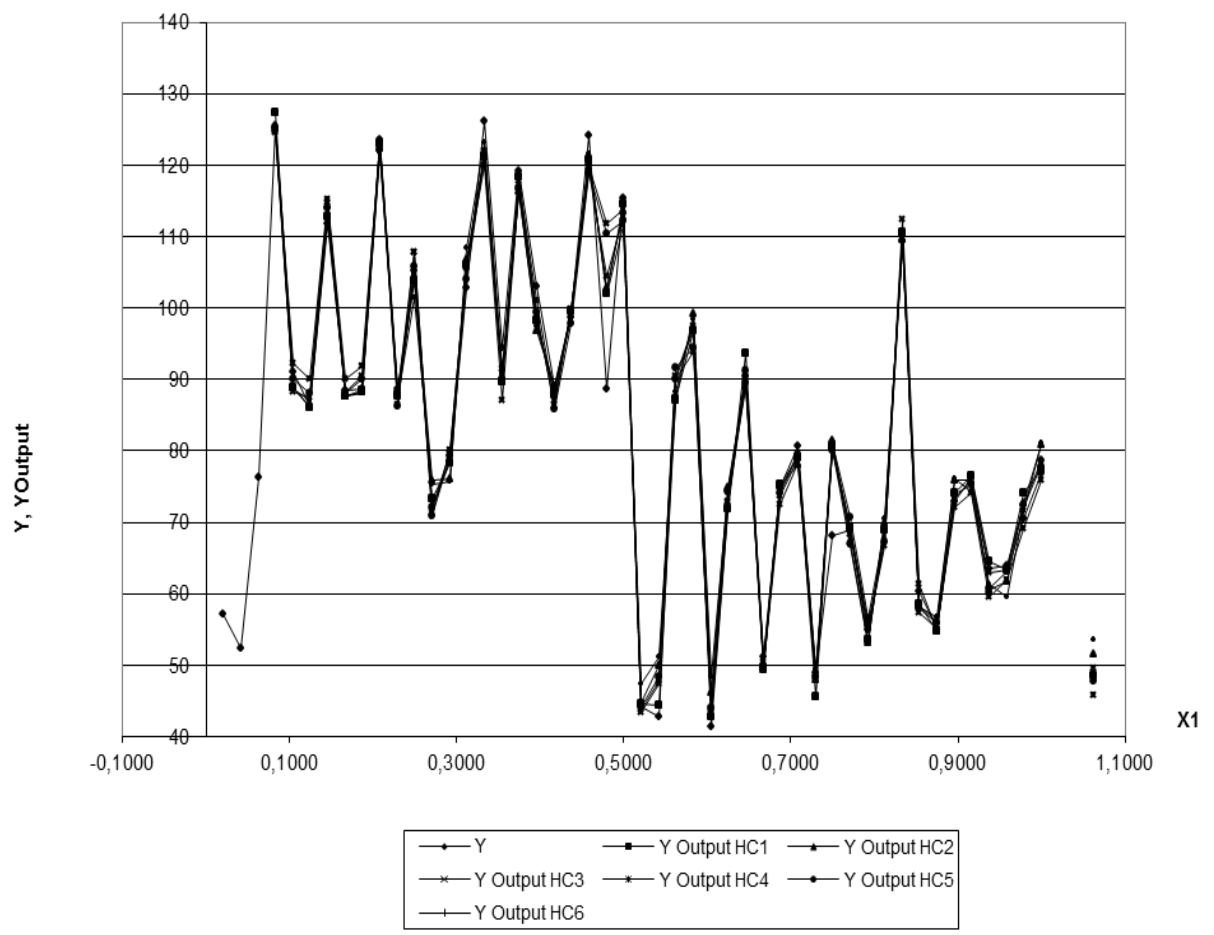

Pис. 2. Экспериментальные Y и расчетные значения Y Oиtрит по всем шести сетям ансамбля в зависимости от времени $X_{1}$

Bulletin of Ural Federal University. Series Economics and Management. 2017. Vol. 16. No 2. PP. 227-245 
ет примерно линейный рост со скоростью в среднем:

$\left(\Delta \hat{Y} / \Delta \mathrm{X}_{2}\right)_{\mathrm{cp}=} 14,7$ млн руб./0,5\% $=29,33$ млн руб./1\%.

То есть при повышении норматива на
$1 \%$ наполнение бюджета увеличивается на 19,33 млн руб.

Подчеркнем, что эта оценка, характерна для существующих «линейных» методик расчета наполнения бюджета и прогноза,

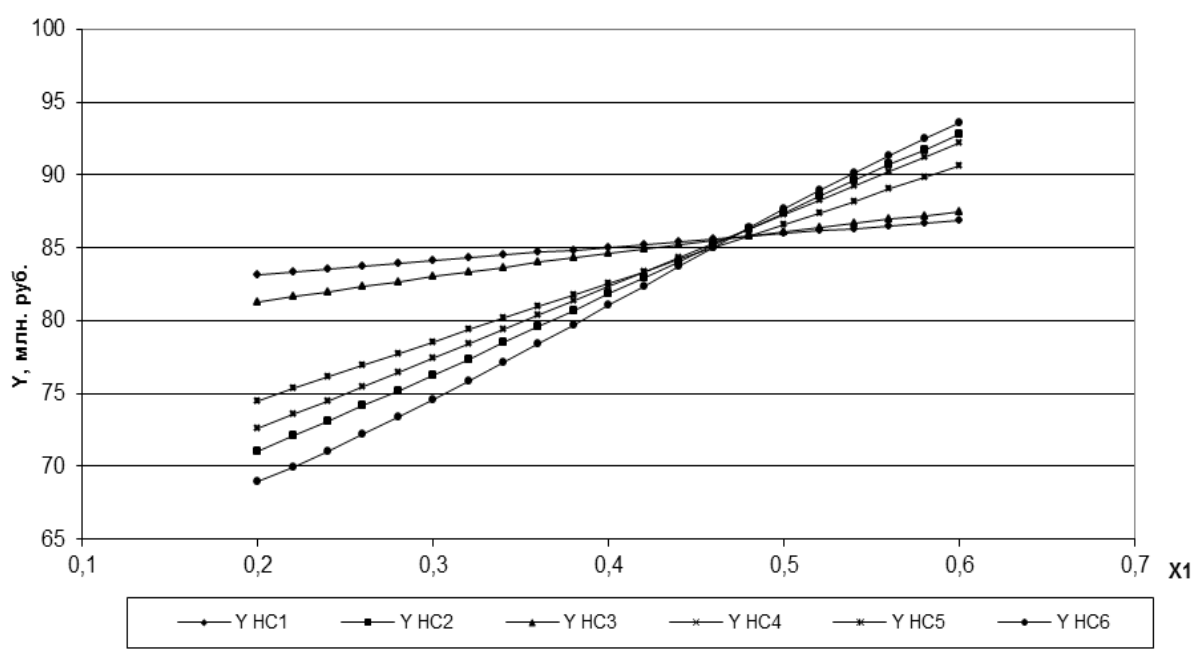

Puc. 3. Y om $X_{1}$

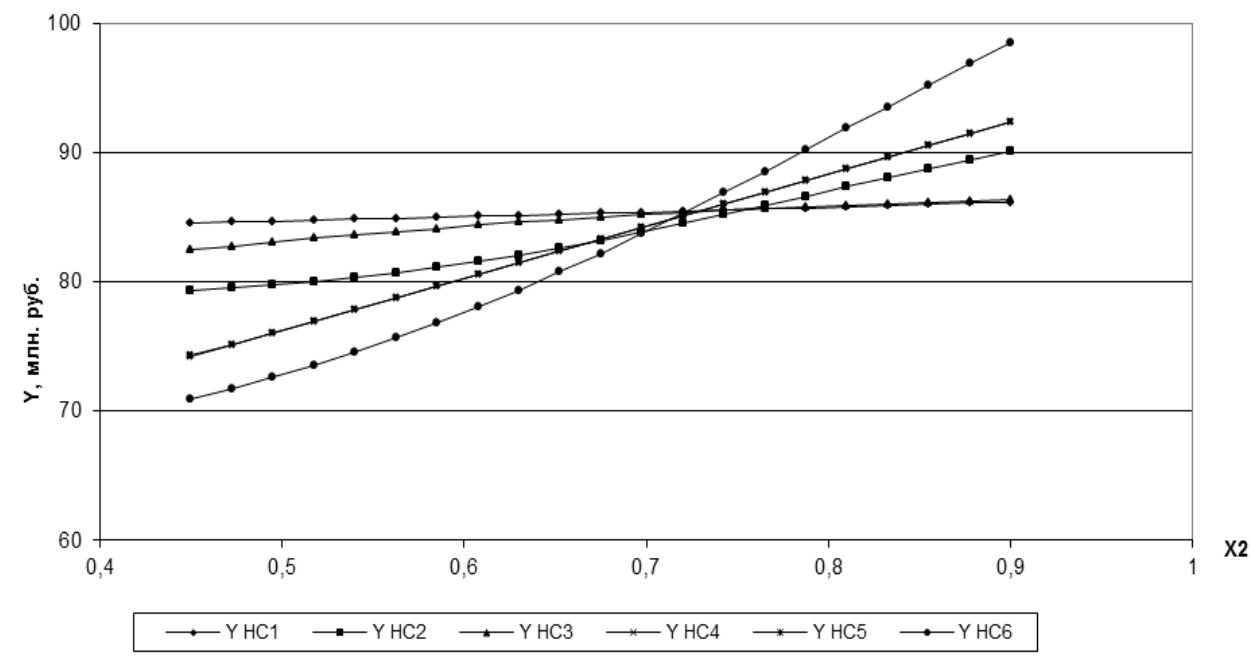

Puc. 3. Y om $X_{2}$ 
также является виртуальной, идеализированной, поскольку не учитывает совместное действие факторов в их сложной динамике по рис. 2. Нейросетевая модель дает реальные приближенные оценки.

3. Зависимость $\hat{Y}$ от фактора $X_{9}$ информация об управленческих расходах по обслуживанию бюджета, в т.ч. фонда оплаты труда (ФОТ) муниципальных госслужащих (рис. 5) дает следующую интересную информацию: рост указанного ФОТ не приводит к росту $\hat{Y}$, а дает в среднем снижение примерно на 2,9 \%, видимо, за счет роста расходов по ФОТ на $37 \%$, что составляет 5 млн руб. Другими словами, увеличивать аппарат госслужащих нецелесообразно.

Таким образом, построенная нейросетевая многофакторная нелинейная прогнозная модель имеет достаточные прогностические свойства по качеству аппроксимации (кривые на рис. 2 практически сливаются).
Такую прикладную модель вполне можно рекомендовать для практики как основу для принятия решений в условиях неопределенности.

\section{Основные выводы}

В данной статье предметом исследования являются обратные задачи восстановления многомерных линейных экономических взаимосвязей, «зашитых» в данных, и смежные задачи ранжирования налогоплательщиков при прогнозировании наполнения местного бюджета. Используется в основном нейросетевой базис аппроксимации многомерных функций. Получаемая модель рассматривается как модель поддержки решений лицом, принимающим решения (ЛПР) по бюджетному администрированию. Условия построения модели крайне тяжелые: данные сильно зашумлены (вплоть до их сознательного искажения), что объясняется стремлением налогоплательщиков в бюджет уменьшить налого-

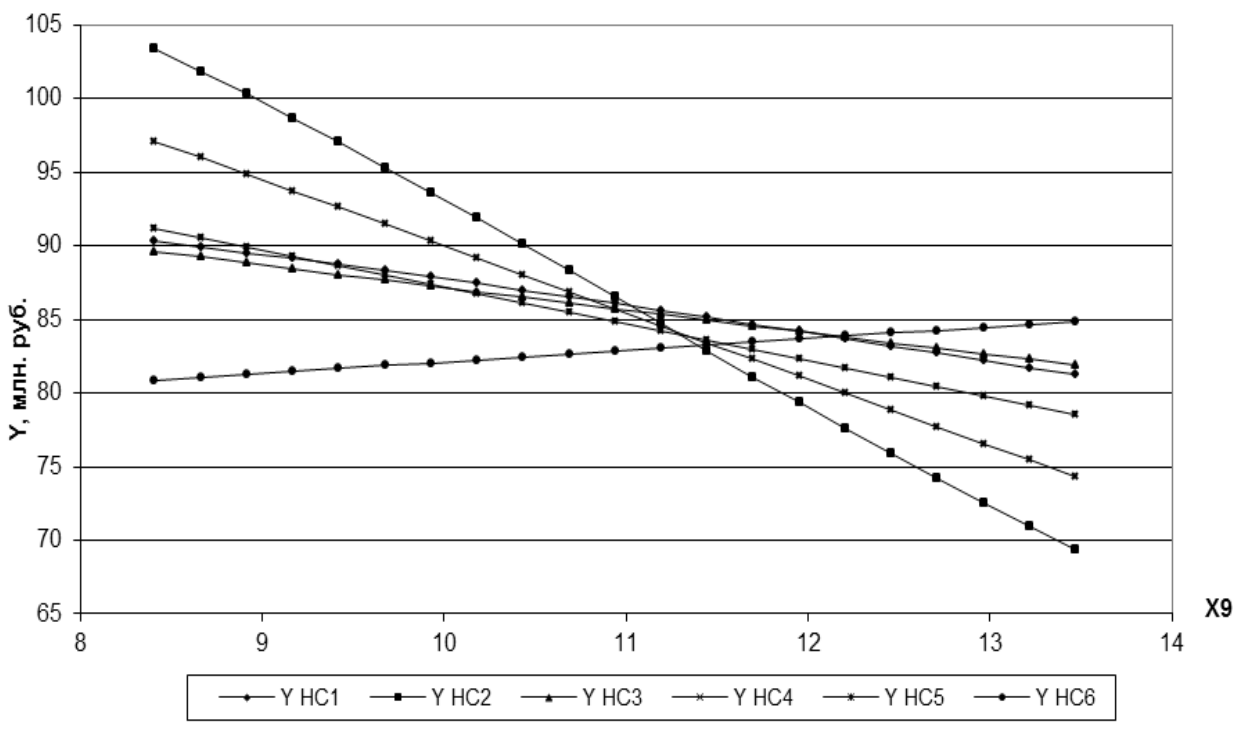

Puc. 3. Y om $X_{9}$

Bulletin of Ural Federal University. Series Economics and Management. 2017. Vol. 16. No 2. PP. 227-245 
облагаемую базу. Зашумление данных усугубляется их дефицитом. Последнее объясняется периодичностью получения информации бюджетными органами - один раз в квартал - и необходимостью кластеризации данных при обучении нейросети при подготовке всех процедур предобработки данных (предрегуляризации).

Математическая модель как инструментарий поддержки решений ЛПР по бюджет- ному администрированию ориентирована на предоставления ему объективной достоверной информации о финансово-экономическом состоянии предприятий-налогоплательщиков, т.е. степени их платежеспособности. Для получения такой информации и предлагается в настоящей статье решение обратной задачи восстановления многомерной нелинейной функции через нейросетевое моделирование.

\section{Список использованных источников}

1. Бирюков А.Н., Гатауллин Р.Ф., Горбатков С.А., Глущенко О.И. Моделирование бюджетных процессов на муниципальном уровне на основе нейросетей. Уфа: Издательство «Восточный университет», 2008. $216 \mathrm{c}$.

2. Бирюков А.Н. Теоретические основы разработки нейросетевых моделей в системе налогового администрирования. Уфа: АН РБ, Гилем, 2011. 380 с.

3. Бирюков А.Н. Бюджетно-налоговое администрирование: нейросетевые методы исследований. Стерлитамак: С.Ф. БашГУ, 2014. 52 с.

4. Горбачев С.В., Сырямкин В.И., Сырямкин М.В., Абрамова Т.В. Нейросетевое моделирование кривой технико-экономического развития в условиях смены технологических укладов // Телекоммуникации. 2014. № 3. С. 40-47.

5. Минц А.Ю. Общие вопросы постановки задач в нейросетевом моделировании // Нейро-нечеткие технологии моделирования в экономике. 2012. № 1. C. 190-206.

6. Тихонов А.Н., Леонов А.С., Ягола А.Г. Нелинейные некорректные задачи. М.: Наука, 1995. 312 с.

7. Хайкин С. Нейронные сети: полный курс / пер. с англ. 2-е изд. М.: Издательский дом «Вильямс», 2006. 1104 с.

8. Окунь А.С., Окунь С.А. Нейросетевое моделирование как инструмент прогнозирования // Финансовая аналитика: проблемы и решения. 2011. №33 (75). C. $45-52$.
9. Ильченко А.Н., Коровин Д.И. Возможности нейросетевого моделирования для совершенствования управленческого учета в системе внутрифирменного бюджетирования // Современные наукоемкие технологии. Региональное приложение. 2007. № 2-3. С. 22-27.

10. Нагоев А.В., Тешев В.А., Блягоз 3.У., Шелехова Л.В. Применение нейросетевых технологий в экономике // Знание. 2016. № 1-1 (30). С. 153-155.

11. Файзрахманов Р.А., Липатов И.Н., Долгова Е.В. Решение задачи идентификации нелинейного дискретного динамического объекта с использованием многослойной нейронной сети // Нейрокомпьютеры: разработка, применение. 2013. № 11. С. 89-95.

12. Ясницкий Л.Н. Интеллектуальные системы. М.: Лаборатория знаний, 2016. $221 \mathrm{c}$.

13. Miroliubova T., Biryukov A. Spatial Differentiation and Market Potential of the Regions: the Case of Russia // Asian Social Science. 2015. Vol. 11, No. 9. P. 96-117.

14. Holvoet T., Valckenaers P. Exploiting the Environment for Coordinating Agent Intentions // Environments for Multi-Agent Systems III, Lecture Notes in Artificial Intelligence. Vol. 4389. Berlin: Springer, 2007. P. 51-66.

15. Xianjun Ni. Research of Data Mining Based on Neural Networks // World Academy of Science, Engineering and Technology. 2008. Vol. 39. P. 381-384. 


\title{
CORRECT FORMULATION OF THE INVERSE PROBLEMS OF RECONSTRUCTION OF MULTIDIMENSIONAL FUNCTIONS USING NEURAL NETWORK MODEL IN BUDGET ADMINISTRATION
}

\begin{abstract}
The object of consideration of this work is very deep penetration of requirements for effective neural network learning algorithms in preprocessing processing. A method has been developed for assessing the adequacy of neural network models in the absence of any a priori information about the distribution law of noise in the data. This is the scientific novelty of this article, as this method provides for interlinked control of the quality of financial data preprocessing and their quality of approximation in the neural network for the budgetary authorities. It is proposed tht the class of problems in budget administration should be considered for which the noise data is quite large, so the need for regularization of hypersurface restoration tasks is a prerequisite of the effectiveness of hybrid models. In particular, the authors show that it is impossible to ignore the incorrect formulation of the problem. To overcome it, there are two ways: introduction of an inverse problem in the class of proper (conditionally correct according to Tikhonov) through additional information about the desired solution, or the control of classical algorithms for solving ill-posed problems. Both ways are based on the achievements of mostly Russian scientists. Existing regularizing algorithms use the original database as some external immutable «Givens». The article uses a meaningful practical example of the proposed systematic approach to the problem of ensuring the stability of neural network mapping when restoring a hypersurface with strong noise data based on the theory of the regularization by $\mathrm{A}$. N. Tikhonov and the Bayesian approach. The essence of this method is that to ensure the viability of the algorithm for the regularization of inverse problems by A. N. Tikhonov base source data is not used as some «over-Stiva» category, and is subjected to pre-processing (structuring) using system-wide laws of Cybernetics (the law of entropy balance of an open system, incomplete suppression dysfunctions of a structured system, redundancy). Thus, the aim of the study is achieved - the essence of the proposed approach is detailed and implemented in the practical concept of «regularization» in this study through the operations of the algorithm in constructing the neural network model.
\end{abstract}

Key words: budget revenues; budget beneficiaries; neural network model; interpretation; tax and nontax revenues; network regularization.

\section{References}

1. Biriukov, A.N., Gataullin, R.F., Gorbatkov, S.A., Glushchenko, O.I. (2008). Modelirovanie biudzhetnykh protsessov na munitsipal'nom urovne

na osnove neirosetei [Application of neural networks to modelling of budget processes at the municipal level]. Ufa, Vostochnyi Universitet.

Bulletin of Ural Federal University. Series Economics and Management. 2017. Vol. 16. No 2. PP. 227-245 
2. Biriukov, A.N. (2011). Teoreticheskie osnovy razrabotki neirosetevykh modelei $v$ sisteme nalogovogo administrirovaniia [Theoretical foundations of the development of neural network models in the system of tax administration]. Ufa, Bashkortostan Academy of Sciences, Gilem Pubising House.

3. Biriukov, A.N. (2014). Biudzhetnonalogovoe administrirovanie: neirosetevye metody issledovanii [Budgetary and tax administration: Neural network research methods]. Sterlitamak, Bashkir State University.

4. Gorbachev, S.V., Syriamkin, V.I., Syriamkin, M.V., Abramova, T.V. (2014). Neirosetevoe modelirovanie krivoi tekhniko-ekonomicheskogo razvitiia $\mathrm{V}$ usloviiakh smeny tekhnologicheskikh ukladov [Neural modelling of the curve of technological and economic development amid technology transition]. Telekommunikatsii (Telecommunications), No 3, 40-47.

5. Mints, A.Iu. (2012). Obshchie voprosy postanovki zadach $\mathrm{v}$ neirosetevom modelirovanii (General questions of tasking in neural network modelling). Neiro-nechetkie tekhnologii modelirovaniia $v$ ekonomike (NeuroFuzzy Modeling Techniques in Economic), No 1, 190-206.

6. Tikhonov, A.N., Leonov, A.S., Iagola, A.G. (1995). Nelineinye nekorrektnye zadachi [Non-linear illposed tasks]. Moscow, Nauka.

7. Haykin, S. (1994). Neural Networks. Macmillan.

8. Okun, A.S., Okun, S.A. (2011). Neirosetevoe modelirovanie kak instrument prognozirovaniia (Neural network modeling as a tool for prediction). Finansovaia analitika: problemy $i$ resheniia (Financial Analytics: Science and Experience), No 33 (75), 45-52.

9. Il'chenko, A.N., Korovin, D.I. (2007). Vozmozhnosti neirosetevogo modelirovaniia dlia sovershenstvovaniia upravlencheskogo ucheta $\mathrm{v}$ sisteme vnutrifirmennogo biudzhetirovaniia (Opportunities for improvement neural network modeling management accounting in the budgeting intercompany). Sovremennye naukoemkie tekhnologii. Regional'noe prilozhenie (Modern High Technologies. Regional Application), No 2-3, 22-27.

10. Nagoev, A.V., Teshev, V.A., Bliagoz, Z.U., Shelekhova, L.V. (2016). Primenenie neirosetevykh tekhnologii v ekonomike (The use of neural network technologies in the economy). Znanie [Knowledge], No 1-1 (30), 153-155.

11. Faizrakhmanov, R.A., Lipatov, I.N., Dolgova, E.V. (2013). Reshenie zadachi identifikatsii nelineinogo diskretnogo dinamicheskogo ob"ekta $\mathrm{s}$ ispol'zovaniem mnogosloinoi neironnoi seti (The solution of problem of identification of nonlinear discrete stationary dynamic object identification by use of a multilayer neural network (MLNN)). Neirokomp'iutery: razrabotka, primenenie [Neural computers: Development, application], No 11, 89-95.

12. Iasnitskii, L.N. (2016). Intellektual'nye sistemy [Intellectual systems]. Moscow, Laboratoriia znanii.

13. Miroliubova, T., Biryukov, A. (2015). Spatial Differentiation and Market Potential of the Regions: the Case of Russia. Asian Social Science, Vol. 11, No. 9, 96-117. 
14. Holvoet, T., Valckenaers, P. (2007). Exploiting the Environment for Coordinating Agent Intentions. Environments for Multi-Agent Systems III, Lecture Notes in Artificial Intelligence, Vol. 4389. Berlin: Springer, 51-66.
15. Xianjun, Ni (2008). Research of Data Mining Based on Neural Networks. World Academy of Science, Engineering and Technology, Vol. 39, 381-384.

\section{Information about the authors}

Biryukov Aleksandr Nikolaevich - Doctor of Economics, Associate Professor, Professor of the Department of Economic Theory and Analysis, Sterlitamak Branch of Bashkir State University, Sterlitamak, Russia (453103, Republic of Bashkortostan, Sterlitamak, Lenin Avenue, 49); e-mail: biryukov_str@mail.ru.

Gluschenko Olga Ivanovna - Candidate of Economic Sciences, Associate Professor, Doctoral Candidate, East Economic-Legal Humanitarian Academy, Ufa, Russia, (450054, Bashkortostan, Ufa, prospect October street, 71/3); e-mail: Olga.glushhenko@mail.ru. 